\title{
Open, randomized, controlled clinical trial of Boswellia serrata extract as compared to valdecoxib in osteoarthritis of knee
}

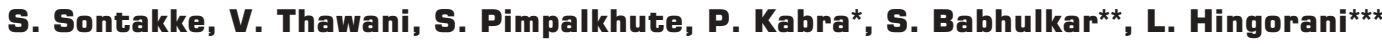

\begin{abstract}
Government Medical College,
*Government Ayurved College,

**Indira Gandhi Government Medical College, Nagpur,

***Pharmanza (India), GIDC, Kansari, India
\end{abstract}

Received: 2.5 .2006

Revised: 27.10 .2006

Accepted: 31.10 .2006

Correspondence to:

V. Thawani

E-mail:

vijaythawani@rediffmail.com

\section{ABSTRACT}

Objective: To compare the efficacy, safety and tolerability of Boswellia serrata extract (BSE) in osteoarthritis (OA) knee with valdecoxib, a selective COX-2 inhibitor.

Materials and Methods: In a randomized, prospective, open-label, comparative study the efficacy, safety and tolerability of BSE was compared with valdecoxib in 66 patients of OA of knee for six months. The patients were assessed by WOMAC scale at baseline and thereafter at monthly interval till 1 month after drug discontinuation. Antero-posterior radiographs of affected knee joint were taken at baseline and after 6 months.

Results: In BSE group the pain, stiffness, difficulty in performing daily activities showed statistically significant improvement with two months of therapy which even lasted till one month after stopping the intervention. In valdecoxib group the statistically significant improvement in all parameters was reported after one month of therapy but the effect persisted only as long as drug therapy continued. Three patients from BSE group and two from valdecoxib group complained of acidity. One patient from BSE group complained of diarrhea and abdominal cramps.

Conclusion: BSE showed a slower onset of action but the effect persisted even after stopping therapy while the action of valdecoxib became evident faster but waned rapidly after stopping the treatment.

KEY WORDS: Anti-inflammatory, herbal medicine, WOMAC.

\section{Introduction}

Osteoarthritis (OA) is a common articular disease. The therapy in modern medicine includes the use of NSAIDs and steroids, which are associated with ADRs and do not reverse the pathological process of the disease.

Boswellia serrata (BS) (Family: Bursaraceae) known as Gaja-bhaksha (implying its ingestion by elephants) in Sanskrit and Sallaki Guggul in Ayurveda is widely used in India. BS gum has been mentioned in the ancient Indian Ayurvedic texts. ${ }^{[1,2]}$ Gum resin extracted from BS possesses good antiinflammatory, anti-arthritic and analgesic activities. ${ }^{[3]}$ It has been claimed to decrease the degradation of glycosaminoglycans thereby helping to prevent the destruction of articular cartilage. ${ }^{[4]}$ The efficacy and safety of BS in OA knee was studied in a randomized, double blind, placebo-controlled trial where BS was reported to be significantly better than placebo in relieving the symptoms of OA. ${ }^{[5]}$ No comparative clinical trial is yet reported with BS. Hence, this study was planned to compare the efficacy, safety and tolerability of BS in OA knee with valdecoxib, a selective COX-2 inhibitor.
The present study was a randomized, prospective, openlabel, comparative trial approved by the Institutional ethics committee. About 66 patients aged between 40 and 70 years of either gender, with primary OA of knee, diagnosed according to the criteria of American College of Rheumatology ${ }^{[6]}$ were recruited from the OPD of Kayachikitsa Government Ayurved College, Nagpur. The diagnosis was based on clinical presentation and X-ray findings and confirmed by the orthopedician. Patients were enrolled between December 2003 and May 2004 and were followed up to 7 months. Patients with history of peptic ulcer, acid peptic disease, concurrent illness, receiving concomitant drug therapy, having history of any drug allergy, having received corticosteroids within four weeks or any anti-inflammatory analgesic three days prior to enrolment, pregnant/lactating women were excluded. Informed, written consent was obtained from those who agreed to participate. Routine hematological investigations were done. The patients were assessed by Western Ontario and McMaster Universities OA index (WOMAC) scale (version VA 3.1) in which decrease in scores suggests improvement ${ }^{|7|}$ and antero-posterior radiographs of affected knee joint in full extension. 
The patients were randomly allocated by SAS system for Windows to two groups of 33 each to receive either Boswellia serrata extract (BSE) (Cap Wokvel ${ }^{\mathrm{TM}}$, manufactured by Pharmanza India) each containing $333 \mathrm{mg}$ of BSE thrice daily (BSE Group) or valdecoxib $10 \mathrm{mg}$ (Tab Valdone, Cadila Pharmaceuticals Limited) once daily (Valdecoxib Group) for six months. The medicines were given orally and the patients were advised to take them after meals. Asking the patients to bring back the empty blister packs during each visit checked the compliance and the consumed drug count was measured. Consumption of $90 \%$ of the drug was considered as adequate compliance. None of the patients became pregnant during the trial period. The age group of study subjects was above 40 years so chances of a patient becoming pregnant were minimal. No advice about contraception was given. The BSE capsule contained the standardized extract of BS gum having minimum $40 \%$ total boswellic acids (BA). The main components of BA in BSE used were 11-keto-beta BA - 6.44\%, 3-0-Acetyl-beta BA-8.58\%, alpha BA -6.93\% and 3-0-acetyl alpha BA-1.853\%. The patients were assessed by WOMAC scale at monthly intervals and at the end of study. Radiograph of the affected knee joint was repeated at the end of study. The patients were under the assigned intervention for 30 days at a time and permitted to continue physiotherapy they were receiving and advised to report ADRs. The drug intervention was for a period of six months and assessment done with WOMAC scale, was repeated at the end of seventh month to evaluate the residual effect of treatment.

WOMAC scores in each group at monthly interval were compared with the basal scores by repeated measures Avova followed by Dunnett's test. Between group comparison was done by unpaired ' $t$ ' test and ' $P$ ' value less than 0.05 was considered statistically significant.

Demographic characteristics of both groups were comparable. Two patients from BSE group and six from valdecoxib group dropped out from the study. From BSE group, one patient dropped due to diarrhea and abdominal cramps and the other due to inadequate control of symptoms. The reason given by all dropouts from valdecoxib group was that symptoms recurred if the patient missed a dose or two.

The basal WOMAC scores for pain, stiffness and difficulty in performing daily activities were compared with the scores at the end of each month. In BSE group, the difference in WOMAC scores was not statistically significant at the end of first month of intervention when compared to baseline $(P>0.05)$. But from second month onwards the decrease was highly significant $(P<0.001)$ as compared to the baseline and remained so throughout the intervention and even one month after the stoppage of the treatment [Table 1].

Six patients from valdecoxib group and seven from BSE group required rescue medication - tablet Ibuprofen $400 \mathrm{mg}$ thrice daily, which was administered till symptomatic improvement.

In valdecoxib group, the decrease in WOMAC scores was statistically significant at the end of one month of treatment as compared to baseline $(P<0.001)$ and persisted as long as the treatment was continued. One month after stopping the treatment, the difference was not statistically significant [Table 1]. Basal WOMAC scores were comparable in the two treatment groups. The difference in WOMAC scores between the two treatment groups was statistically significant at the end of one month for all the three symptoms. The WOMAC Scores with BSE were significantly lower $(P<0.001)$ than with valdecoxib at the end of 7 months for all the three parameters.

There was no difference in the pre- and post-drug radiographs of the affected knee joint. Three patients from BSE group and two from valdecoxib group complained of acidity. They responded to treatment with tablet ranitidine $150 \mathrm{mg}$ twice daily for seven days and chose to continue with the trial.

In the BSE treated group the WOMAC scores did not show any significant decrease after the first month of intervention but from second month onwards became significantly lower as compared to the baseline and continued to be significantly lower till one month after stopping the treatment. This indicates

Table 1

Comparison of WOMAC scores in the two treatment groups at different time intervals

\begin{tabular}{|c|c|c|c|c|c|c|c|c|c|c|}
\hline \multirow{2}{*}{\multicolumn{2}{|c|}{ Time }} & \multicolumn{4}{|c|}{ Pain } & \multicolumn{3}{|c|}{ Stiffness } & \multicolumn{2}{|c|}{$\begin{array}{c}\text { Difficulty in performing } \\
\text { daily activities }\end{array}$} \\
\hline & & \multicolumn{2}{|c|}{$\begin{array}{c}\text { Boswellia serrata } \\
\text { extract }\end{array}$} & \multicolumn{2}{|c|}{ Valdecoxib } & \multicolumn{2}{|c|}{$\begin{array}{c}\text { Boswellia serrata } \\
\text { extract }\end{array}$} & Valdecoxib & $\begin{array}{c}\text { Boswellia serrata } \\
\text { extract }\end{array}$ & Valdecoxib \\
\hline \multicolumn{2}{|l|}{ Base-line } & \multicolumn{2}{|c|}{$245.3 \pm 77.57$} & \multicolumn{2}{|c|}{$246.0 \pm 71.38$} & \multicolumn{2}{|c|}{$87.67 \pm 36.30$} & $91.45 \pm 22.87$ & $893.3 \pm 220.7$ & $877.8 \pm 156.6$ \\
\hline \multicolumn{2}{|c|}{ After 1 month } & \multicolumn{2}{|c|}{$225.5 \pm 85.94^{*}$} & \multicolumn{2}{|c|}{$140.2 \pm 74.12^{\#}$} & \multicolumn{2}{|c|}{$78.55 \pm 38.29^{*}$} & $47.76 \pm 27.79^{\#}$ & $824.7 \pm 269.6^{*}$ & $434.2 \pm 271.8^{\#}$ \\
\hline \multicolumn{2}{|c|}{ After 2 months } & \multicolumn{2}{|c|}{$159.5 \pm 96.58^{\#}$} & \multicolumn{2}{|c|}{$152.2 \pm 193.4^{\#}$} & \multicolumn{2}{|c|}{$57.91 \pm 34.24^{\#}$} & $39.55 \pm 23.18^{\#}$ & $582.7 \pm 296.2$ & $348.7 \pm 207.5^{\#}$ \\
\hline \multicolumn{2}{|c|}{ After 3 months } & \multicolumn{2}{|c|}{$128.6 \pm 84.56^{\#}$} & \multicolumn{2}{|c|}{$105.2 \pm 59.61^{\#}$} & \multicolumn{2}{|c|}{$47.48 \pm 31.05^{\#}$} & $33.70 \pm 21.10^{\#}$ & $483.6 \pm 274.7$ & $330.5 \pm 205.3^{\#}$ \\
\hline \multicolumn{2}{|c|}{ After 4 months } & \multicolumn{2}{|c|}{$107.8 \pm 79.57^{\#}$} & \multicolumn{2}{|c|}{$101.9 \pm 71.04^{\#}$} & \multicolumn{2}{|c|}{$41.06 \pm 29.10^{\#}$} & $34.58 \pm 24.15^{\#}$ & $408.7 \pm 262.1$ & $335.9 \pm 229.7^{\#}$ \\
\hline \multicolumn{2}{|c|}{ After 5 months } & \multicolumn{2}{|c|}{$88.3 \pm 65.36^{\#}$} & \multicolumn{2}{|c|}{$82.12 \pm 63.39^{\#}$} & \multicolumn{2}{|c|}{$33.73 \pm 23.58^{\#}$} & $27.61 \pm 22.05^{\#}$ & $348.7 \pm 219.7$ & $269.6 \pm 212.3^{\#}$ \\
\hline \multicolumn{2}{|c|}{ After 6 months } & \multicolumn{2}{|c|}{$82.85 \pm 62.31^{\#}$} & \multicolumn{2}{|c|}{$85.42 \pm 68.85^{\#}$} & \multicolumn{2}{|c|}{$30.45 \pm 21.84^{\#}$} & $29.18 \pm 24.14^{\#}$ & $325.2 \pm 207.9$ & $282.8 \pm 225.3^{\#}$ \\
\hline \multicolumn{2}{|c|}{ After 7 months } & \multicolumn{2}{|c|}{$81.67 \pm 63.29^{* *, \#}$} & \multicolumn{2}{|c|}{$197.7 \pm 111.3$} & 29.82 & $1.55^{* *, \#}$ & $81.15 \pm 41.49$ & $322.6 \pm 207.8^{* *, \#}$ & $775.5 \pm 392.4$ \\
\hline Repeated & $\mathrm{F}$ & 26.62 & 3.850 & 68.02 & 11.42 & 111.7 & 10.07 & & & \\
\hline $\begin{array}{l}\text { Measures } \\
\text { ANOVA, }\end{array}$ & $d f$ & 7,263 & 7.263 & 7,263 & 7,263 & 7,263 & 7,263 & & & \\
\hline
\end{tabular}


that BSE has slower onset of action and takes more than a month for the action to manifest. At the same time its effect was persistent even after one month of stoppage of treatment. This is a valuable finding as most of the currently used drugs in OA from modern medicine provide short-lasting symptomatic relief, as also seen by us in the valdecoxib group, where the onset of action was fast but waned rapidly on stoppage of the treatment.

Studies report that BSE prevented the degradation of articular cartilage, ${ }^{[5]}$ which could be one of the mechanisms in arresting the progression of $\mathrm{OA}$. A possible reason of the persistent effect of BSE even one month after stopping treatment may be an effect on the underlying pathology in OA. This is a significant finding since none of the existing anti-inflammatory medicines affects the basic pathology in OA. It is clinically difficult to study the effect of a drug on articular cartilage and can only be attempted with arthroscopy. We had planned to perform arthroscopy in five patients from each group, but none of the study participants gave willingness to undergo this invasive investigation when the method and purpose was explained to them. Though the WOMAC scale that we used is an internationally accepted, it is a subjective scale. This is a modified visual analogue scale which consists of questions based on 3 symptoms viz. pain, stiffness and difficulty in performing physical activity. For each question the patient has to mark on a scale between 0 and 100. Say for pain 0 indicates no pain and 100 is maximum pain. Adding up the scores of all the questions for a particular symptom gives total score for that symptom. Decrease in score suggests symptomatic improvement.

A 32 week randomized, placebo controlled clinical evaluation of a polyherbal combination containing BSE in OA of the knee reported it to be significantly better than placebo, without any significant adverse effects. ${ }^{[8]}$ In our study also no severe side effects were reported with BSE.

Reports show that BSE inhibited human leucocyte elastâse, which could be of help in autoimmune disorders like rheumatoid arthritis. ${ }^{[9]}$ The gum resin of BS was shown to block leukotriene biosynthesis due to the action of BA constituents. ${ }^{[10]}$ The BAs inhibited lipoxygenase (LO) synthesis via 5-LO, but did not affect the 12-LO and cyclo-oxygenase (CO) activities. BAs specifically decreased the synthesis of proinflammatory 5-LO products like 5-hydroxyeicosatetraenoic acid and LT-B4. ${ }^{[11]}$

One patient dropped out of the study after one week due to diarrhea. Whether diarrhea was due to BSE or other causes is difficult to predict. There were no serious ADRs with valdecoxib in our study but the recent reports of valdecoxib producing serious cardiovascular and dermatological toxicity are of concern. ${ }^{[12,13]}$ We included valdecoxib as a comparator drug because at the time of protocol submission it was the latest drug on the scene, which was being widely prescribed for OA. However, subsequently it has been banned in India due to serious ADRs.

Thus, in terms of safety, efficacy and duration of action, the present study shows that BSE was superior to valdecoxib, except for the slower onset of action compared to valdecoxib.

\section{Acknowledgement}

We are grateful to Mrs. K. J. Gharpure, Research Pharmacist, Pharmacology Department, Government Medical College, Nagpur for internally peer-reviewing the manuscript and Dr. G. Dakhale and Dr. A. Turankar, ex-Lecturers in Pharmacology, Government Medical College Nagpur for helping in statistical analysis.

\section{References}

1. Boswellia serrata. Altern Med Rev 1998;3:306-7.

2. Kulkarni RR, Patki PS, Jog VP, Gandage SG, Patwardhan B. Treatment of osteoarthritis with a herbomineral formulation: A double-blind, placebocontrolled, cross-over study. J Ethnopharmacol 1991;33:91-5.

3. Selected medicinal Plants of India -A Monograph of identity, safety and clinical usage. Compiled by Swami Prakashanand Ayurved Research Centre (SPARC) for Chemixil. Tata Press: India; 1992. p. 65-6.

4. Reddy GK, Chandrakasan G, Dhar SC. Studies on the metabolism of glycosaminoglycans under the influence of new herbal anti-inflammatory agents. Biochem Pharmacol 1989;38:3527-34.

5. Kimmatkar N, Thawani V, Hingorani L, Khiyani R. Efficacy and tolerability of Boswellia serrata extract in treatment of Osteoarthritis of knee- a randomized, double blind, placebo controlled trial. Phytomedicine 2003;10:3-7.

6. Hart DJ, Spector TD. The classification and assessment of Osteoarthritis. Baillieres Clin Rheumatol 1995;9:407-32.

7. Nicholas B. WOMAC Osteoarthritis Index VA 3.1. User Guide 5. University of Queensland Faculty of Health Sciences: Australia; 2002. p. 15-6.

8. Chopra, A, Lavin P, Patwardhan B, Chitre D. A 32-week randomized, placebocontrolled clinical evaluation of RA-11, an ayurvedic drug, on osteoarthritis of the knees. J Clin Rheumatol 2004;10:236-45.

9. Safayhi H, Rall B, Sailer ER, Ammon HP. Inhibition by Boswellic acids of human leucocyte elastase. J Pharmacol Exp Ther 1997;281:460-3.

10. Ammon HP, Mack T, Singh GB, Safayhi H. Inhibition of leukotriene B4 formation in rat peritoneal neutrophils by an ethanolic extract of the gum resin exudate of Boswellia serrata. Planta Med 1991;57:203-7.

11. Ammon HP, Safayhi H, Mack T, Sabieraj J. Mechanism of antiinflammatory actions of curcumine and boswellic acids. J Ethnopharmacol 1993;8:113-9.

12. Talhari C, Lauceviciute I, Enderlein E, Ruzicka T, Homey B. COX-2 selective inhibitor, Valdecoxib induces severe allergic skin reactions. J Allergy Clin Immunol 2005;115:1089-90.

13. Young D. FDA ponders future of NSAIDs: Pfizer reluctantly withdraws Bextra. Am J Health Syst Pharm 2005;62:997-1000.

\section{Announcement}

\section{Dr. J. C. Patel Birth Centenary Celebration Committee}

The year 2008 is the Birth Centenary Year of Dr. J. C. Patel. Some of his students/admirers felt that it would be a good idea to celebrate this Centenary Year by organizing CMEs, Orations/Lectures, Conferences, etc during the year. He was associated with many professional bodies, which meet regularly every year; during these annual meetings/conferences, a lecture/ symposium, etc. can be organized as a part of Centenary celebrations. We would like to form a Dr. J. C. Patel Birth Centenary Celebrations Committee. All his past students/admirers are invited to join the committee (without any financial commitment). Kindly communicate your name, designation, postal address, telephone number and E-mail: to Dr. B. C. Mehta at Flat 504, Prachi Society, Juhu-Versova Link Road, Andheri (W0, Mumbai - 400053 (drmehta.bc@gmail.com). 